\title{
Hurried Psychiatric Labelling: A Preventable Medical Error in an Emergency Department
}

\author{
Mohammad Javad Behzadnia ${ }^{1^{*}}$ \\ ${ }^{1}$ Trauma Research Center, Baqiyatallah University of Medical Sciences, Tehran, Iran \\ *Corresponding Author: Mohammad Javad Behzadnia, Assistant Professor, Trauma Research Center, Baqiyatallah \\ University of Medical Sciences, Nosrati Alley, Sheikh Bahaie St, Molla Sadra St, Vanaq sq., Tehran, Iran. Tel: +98- \\ 2181262012,Email: Behzadnia@bmsu.ac.ir
}

Received January 18, 2019; Accepted March 6, 2019; Online Published April 22, 2019

\section{Dear Editor,}

Because societies today are industrialized, physical and psychiatric problems have increased significantly. Simple medical approaches are no longer sufficient for managing patients. This is quite obvious in an overcrowded emergency department. Moral and legal aspects of patient management should also be considered. Emergency management is like a double-edged sword. Physicians encounter both somatic and psychiatric presentations simultaneously. In such situations, decision-making can be difficult. ${ }^{1,2}$ Immediately marking a patient as a histrionic or conversion case is a wrong approach that is currently seen in some emergency departments.

Recent forensic medicine cases have explained some clues; life-threatening conditions that had not been given much attention by the physician; some were even considered as psychiatric cases. This is more important when an organic problem is hiding behind a psychiatric presentation (pseudo-psychiatric emergencies) and could easily be ignored or even mismanaged.

Overcrowding and full-stress situations are the two main factors leading to labeling errors. Normal adaptive responses to stress are due to the sympathetic nervous system, adrenal or cortisol response; an abnormal or improper psychiatric response may indicate organic problems rather than a purely psychiatric disorder. ${ }^{3}$ Sometimes, the conversion disorder may present immediately after a medical procedure such as surgery; hence, it is necessary to rule out critical complications rather than tagging the patient initially as a psychiatric case. ${ }^{4}$

Experimental studies and expert opinions explain many critical conditions in which an erroneous first emergency impression has led to catastrophic outcomes; medical problems such as acute myocardial infarction, pulmonary emboli, aortic dissection, and serious brain hemorrhages could display as some psychiatric presentations like anxiety, agitation, unusual behavior, and so on. Therefore, encountering suspicious cases requires an initial assessment to rule out critical somatic risks. Furthermore, such kind of problem should be determined based on psychiatric (DSM-V) criteria. $^{5}$

Accordingly, it is strongly recommended that an accurate history should be taken and a physical examination conducted before a patient is labeled as a psychiatric case. In new cases, even with evidence of a psychiatric issue in an emergency department, further assessment is advised in the future.

\section{Conflict of Interest Disclosures}

The author declares that he has no conflicts of interest..

\section{Ethical Approval}

Not applicable.

\section{References}

1. Purcell TB. The somatic patient. Emerg Med Clin North Am. 1991;9(1):137-159.

2. Dworetzky BA, Weisholtz DS, Perez DL, Baslet G. A clinically oriented perspective on psychogenic nonepileptic seizurerelated emergencies. Clin EEG Neurosci. 2015;46(1):26-33. doi:10.1177/1550059414566880.

3. Testa A, Giannuzzi R, Sollazzo F, Petrongolo L, Bernardini L, Daini S. Psychiatric emergencies (part I): psychiatric disorders causing organic symptoms. Eur Rev Med Pharmacol Sci. 2013;17 Suppl 1:55-64.

4. Siket MS, Merchant RC. Psychogenic seizures: A review and description of pitfalls in their acute diagnosis and management in the emergency department. Emerg Med Clin North Am. 2011;29(1):73-81. doi:10.1016/j.emc.2010.08.007.

5. Vermeulen $\mathrm{M}$, Willems $\mathrm{MH}$. Conversion disorder: from DSM IV to DSM 5 or from a psychiatric to a neurological diagnosis. Tijdschr Psychiatr. 2015;57(8):569-576. [Dutch]. 